the bowel was exactly similar in appearance to the part shown in the annexed figure. It will be observed that the surface of the mucous membrane is thickly beset with small polypoid growths, the largest of which are about half an inch in length. The majority of the growths are pear-shaped or ovoid, the narrow end being the one by which the growth is attached to the mucous membrane.

Microscopic examination shows that each growth contains a central core of vascular connective tissue, which is covered by a thickish stratum of adenoid tissue with many tubular glands similar to those normally present in the intestinal mucous membrane, but not quite so regular, and separated by a larger amount of interglandular tissue.

\title{
THE SUSCEPTIBILITY OF THE ASS TO TUBERCULOSIS.
}

By the Same.

THE following note may be of interest when read in connection with the one on the same subject by $\mathrm{Mr}$ Stewart Stockman, which appears at an earlier part of this number of the Joumal.

On the 22nd January last I injected into the right jugular vein of a healthy donkey $2 \mathrm{cc}$. of a liquid rich in tubercle bacilli. The animal was an aged castrated male, and the liquid used for inoculation was prepared by rubbing up a quantity of caseo-purulent matter from the tuberculous spleen of a horse (natural case of the disease) with sterile water. The coarser particles were allowed to settle, and a cover-glass preparation then made from the liquid showed tubercle bacilli in every field of the microscope.

The normal temperature of the donkey was between $99^{\circ}$ and $100^{\circ}$, and between the date of inoculation and the $15^{\text {th }}$ February the highest temperature recorded was IOI $6^{\circ}$ (27th January).

On the I $5^{\text {th }}$ February at 9 a.m. the donkey was tested with tuberculin, the quantity injected being $2 \mathrm{cc}$. The result was as follows :-

Hour.

9 a.m.
3 p.m.
6 p.m.
9 p.m.
I 2 midnight
Temperature.

$98 \cdot 2$

$100^{*} 3$

$104^{\circ} 4$

104.2

$104^{\circ} \mathrm{O}$

At 3 p.m. on the following day the temperature was $102^{\circ} 2^{\circ}$, but on the 17 th February it had fallen to $997^{\circ}$. From the latter date till the 22nd March it only once reached IOI ${ }^{\circ}$, viz., on the $\mathrm{I} 8$ th March, when it was $1022^{\circ}$.

At 9 a.m. on the 22 nd March the donkey was again tested with tuberculin, with the following result:-

Hour.

9 a.m. .

3 p'm. .

6 p.m. .

9 p.m.

I 2 midnight
Temperature.

$99^{\circ} 4$

IOO 4

IOI 6

ÍO2'O

IOI 8 
On the following day the temperature was $1024^{\circ}$, but on the $24^{\text {th }}$ March it had again become normal $\left(99^{\circ} 8^{\circ}\right.$.

The animal was subjected to a third test on the I2th May, at 9 a.m., with the following result :- -

Hour.

9 a.m.

3 p.m.

6 p.m. .

9 p.m.

12 midnight
Temperature.

IOI'3

99.8

IOI' 2

IOI 4

$100^{\circ} \mathrm{O}$

On this occasion, therefore, there was no reaction.

The test was repeated at 9 a.m. on the $23 \mathrm{rd}$ June, and on that occasion the temperatures recorded were as follows :-

Hour.

9 a.m.

3 p.m. .

6 p.m. .

9 p.m. .

I2 midnight .
Temperature.

100.3

$1000^{\circ}$

IOI'3

$102 \%$

$100 \% 8$

The result in this case appeared a little doubtful, but it is very probable that the slight rise at the ninth and twelfth hours was accidental. At any rate it was not sufficient to indicate that the donkey was tuberculous.

The animal was killed on the 24th June, and the post-mortem examination revealed no trace of tuberculous disease anywhere.

The interpretation which must be placed on the facts above recorded is that the intravenous inoculation practised on the 22nd January did determine a temporary infection; and in confirmation of this it may be mentioned that for some weeks after the 15 th February the animal's respiratory movements were abnormally frequent.

One might have supposed that, if the ass species is susceptible to tuberculosis, experimental infection would be obtained with most certainty by using bacilli from the horse, as the most nearly related species. It is very doubtful, however, whether there is any general law of that kind. It appears to be more probable that, whatever be the usual source of the tubercle bacilli with which horses are occasionally naturally infected, the bacilli found in the lesions at the time when the horse succumbs to tuberculosis are less virulent for all animals than those found in cases of bovine tuberculosis. At any rate, in this instance there is other evidence besides that derived from the post-mortem of the donkey to prove that the equine bacilli were of comparatively low virulence. Simultaneously with the donkey, on the 22nd January, a rabbit and a two-year-old heifer were inoculatcd intravenously with the same emulsion of tubercle bacilli, the former receiving $\frac{1}{2} \mathrm{cc}$. and the latter $2 \mathrm{cc}$.

The rabbit died from tuberculosis on the gth April last, eleven weeks after infection.

About a fortnight after the intravenous inoculation the heifer's temperature began to rise, and on the 7 th February it was $106^{\circ}$. At the same time the animal began to lose condition, became dull, and 
.its respirations increased in frequency. On the 3 rd March, at 9 a.m., the heifer was tested with tuberculin, and the result was as follows:-

Hour.

9 a.m.

3 p.m.

6 p.m.

9 p.m.

I 2 midnight .
Temperature.

IO2.6

$107^{\circ} \mathrm{I}$

$107 \cdot 9$

$105 \cdot 6$

I04. 4

Subsequently the symptoms pointing to the existence of a miliary tuberculosis of the lungs declined, and at the present time the animal is in excellent condition, and to all outward appearance perfectly healthy. Nevertheless, unlike the donkey, it reacted distinctly to tuberculin when tested on the $23 \mathrm{rd}$ June. To add to the interest of this case, it has lately become evident that the heifer is pregnant, although this was not suspected at the time when it was inoculated.

\section{A CASE OF TUBERCULOSIS IN A SHEEP.}

By the Same.

IN view of the rarity of tuberculosis among British sheep, it is a little remarkable that two cases of the kind should fall to be recorded in this number of the Journal. At page $102 \mathrm{Dr}$ Foulerton describes a case which recently came under his notice, and the following are the particulars of another case.

On the 25th March last Mr King, Chief Veterinary Inspector to the Corporation of London, sent for my inspection portions of the viscera of a sheep, and subsequently. I learned from $\mathrm{Mr}$ King that the animal in question was a four-year-old crossbred ewe, which had been killed in London. The notes made regarding the parts forwarded to me were as follows :-

The lung sent is to a large extent destroyed and replaced by completely caseous and partially calcified material, the diseased areas varying from the size of a pea to that of a pigeon's egg. The bronchial glands are enlarged to the volume of a hen's egg, and similarly caseous and calcified. The mediastinal gland is in a similar condition, and it is nearly as thick as one's wrist. Attached to a piece of the diaphragm there is a portion of the spleen, with numerous caseous tumours in it, one of them being as large as a turkey's egg. The greater part of the liver is present, and that also contains numerous caseous nodules, none of which are larger than a hazel nut. The gastro-hepatic omentum contains what appears to be a number of enlarged lymphatic glands, each about the size of a pigeon's egg. Only one kidney is sent, and it shows under the capsule a yellowishwhite tubercle about the size of a barley grain.

Cover-glass preparations were made from the lung lesions and from the caseous mediastinal gland, but no tubercle bacilli were thus detected. Tubes of solid blood serum were inoculated from the lung lesions, and two guinea-pigs were also inoculated subcutaneously, one from the liver and the other from the lung.

After three weeks' incubation a cover-glass preparation made with 